\title{
Is it Possible to Have Meaningful Phase III Clinical Trial? Establishing Level, I Evidence May Be Counter-Productive
}

\author{
Debnarayan Dutta*, Ramkrishna Kamath and Sruthi K \\ Department of Radiation Oncology, Amrita Institute of Medical Science, Kochi, India \\ *Corresponding Author: Debnarayan Dutta, Head- Radiation Oncology, Amrita Institute of Medical Science, Kochi, India.
}

Received: July 29, 2019; Published: October 01, 2019

DOI: $10.31080 /$ ASMS.2019.03.0429

All treatment protocols should be based on 'well-designed phase III clinical studies' and need long-term follow up in phase-IV studies (in clinical practice). These treatment protocols will give us the expected outcome details and probable toxicity profile as well. However, in oncology practice many a times this is not followed. There are a few examples where 'alert' of a new treatment option was done and approval of treatment was based on very short and inadequate study. These practices sometimes have boomeranged the medical fraternity. And in a few situations, the 'alert' was reverted in the opposite direction - as 'to not to use the treatment modality'!!

Thalidomide is a classical example of such an early and misdirected euphoria. Thalidomide was approved for pregnancy induced vomiting, based on a small short follow-up study. Thalidomide was no doubt a very potent anti-emetic medicine for pregnant women. Hence, Thalidomide became a popular 'antiemetic' in pregnant women, and only after several reports of short extremity babies or 'thalidomide babies', the drug was withdrawn in a haste. Thalidomide is a prototype case of fallacies in approval without long-term follow up. Though many laws were made after this disaster to stop such incidence, unfortunately we still get this kind of 'alert', sometimes with similar fate! Proton therapy in lung cancer is an example. There was tremendous amount of euphoria and excitement regarding lung cancer treatment with proton therapy as dose escalation would improve local control and 'stiff dose fall off' would improve toxicity profile. Theoretical and dosimetry superiority may push many clinicians to treat lung cancer with Proton therapy. Only a randomized study proved inferiority of proton over photon and then a Pandora's box of treatment delivery uncertainties was exposed.
Sometime, there is role reversal as well. In today's era, phase III studies are in another extreme position. Unfortunately, this position may not be better than the 'Thalidomide era', where clinical trials were more convenient. In this era, 'ultra-guarded' randomized trials are the norm to avoid any conflict. This approach may be doing similar harm like the Thalidomide era.

In early lung cancer, there is a debate between surgery or radiosurgery as the treatment of choice. In stage T1 - 2 disease, surgery is considered the treatment of choice. But there is no randomized study comparing with other modalities. In stage III - IV, radiation along with chemotherapy takes the maximum treatment responsibility and surgery is usually not an option. In recent years even in early lung cancer (stage 1), radiosurgery has put up a real challenge against 'age old' surgery as an option. Phase 2 studies with radiosurgery are very promising. Randomised study comparing surgery and radiosurgery would have been ideal and can sort out the dilemma of the best treatment option in early lung cancer. Now the real problem is, if surgery is proven to be inferior, then the role of cardio-thoracic surgery (VATS) in lung cancer would be severely compromised. On the other hand, if radiosurgery is proven to be inferior then jobs of 'radio-surgeons' specialized in lung cancer treatment would be under serious threat. Three randomized studies started (STAR, ROSEL) and were stopped due to poor accrual!!! The question remains unanswered, thus securing the job of the surgeons and radiation oncologists, at least till a level I evidence is available! However, the best treatment for early lung cancer still remains undecided.

Similarly, in low and intermediate risk prostate cancer there is always a debate regarding surgery and 'ultra-hypo fractionated' radiation or radiosurgery as a treatment option. Unfortunately, there 
is no ongoing randomized study comparing surgery to radiosurgery in this patient cohort. Neither is anyone interested in doing it. Thousands of patients in the cohort are treated routinely with either surgery or radiosurgery. So, at present as there is no level I evidence, early prostate cancer patients are treated with radiation therapy if they meet a radiation oncologist or get surgery only as an option, if they meet a surgeon. The treatment option is not based on evidence, but the fate of the patient! Interestingly, in the last two decades there are hundreds of studies comparing different radiation therapy treatment schedules - short course, long-course, ultra-short course. Though they are all bio-equivalent dosage schedules and we don't expect a difference in outcome the studies have been done. But not one study answers the basic question of surgery or radiation 'in early prostate cancer. In reality, we do not want to know the answer. We wish to plan 'clinical trials' based on our obligations and virtues and not on real scientific questions. Instead of focusing on a theoretical change in few points of IQ function or quality of life score between two treatment types, the need of the hour is to know our best treatment option.

Till the last century, surgery was the only treatment option in laryngeal cancers with sacrifice of the voice box. In 1990s, Veteran trials group conducted randomized study with 'organ preservation' approach in laryngeal cancers. This landmark study helped two-third (63\%) of laryngeal cancer patients to save their voice. This was a definitive landmark study that changed the lives of many people. Similar landmark study was on breast preserving surgery - comparing preservation of breast and mastectomy with end point as similar outcome. Since then millions of women have had breast conservation and also preserved their body image function.

In recent times, there is a perfect example as to why we do not look for genuine answers of a valid and pertinent question. Role of targeted therapy (cetuximab) in head and neck cancer was proven in a 20 year old randomized study (Bonners study). The study design was flawed, as radiation plus cetuximab was compared with radiation alone and not with radiation and chemotherapy (cisplatin). This Bonners study $(n=416)$ showed benefit of using cetuximab along with radiation therapy in head and neck cancer. Cetuximab was also a 'standard' of care, courtesy this study. However, still a large number of head and neck cancer patients were receiving cisplatin along with radiation therapy and not cetuximab. A new trial comparing cisplatin and cetuximab was required to establish cetuximab as 'the' option in this patient cohort. Well-designed multi-centric study (De - ESCALaTE study, $\mathrm{n}=334$ ) comparing chemotherapy and targeted therapy (cetuximab) was done in oropharyngeal cancers with HPV positive subgroup in which cetuximab was supposed to be the most effective. The result was shocking as patients with cetuximab had poorer survival (2 - yr OS $89.4 \%$ vs $97.5 \%$ ), higher local recurrence rate (16\% Vs 6\%) and no difference in toxicity. Hence, after the study cetuximab is not considered an option in early or locally advanced head and neck cancer. If this study was not conducted, we would have still used cetuximab in many head and neck cancers assuming it to be good for the patient with less side effects. This study opened our eyes and forced us to realize that whatever we see inlow-level evidence studies, needs deep introspection before implementation. We may use them for our justification or rationalization of treatment, but unfortunately it may not be of real benefit to anyone.

Now a days, majority of the randomized studies are comparing different high-end molecules expecting survival benefit of few days or weeks. The clinical relevance of these studies and how much they will help the patients poses a real dilemma. Definitively in most situation these newer medicines are not cost effective. Unfortunately, we will see these kinds of studies only in the near future. And the blame lies with us. We do not want to get an answer for the real question. Our fear of losing out if our speciality becomes irrelevant, our fear of becoming irrelevant as a specialist holds us back from asking the truth. But the 'real truth' is that no modality will be irrelevant if it is helping the patients. And no modality that is not helping the patients can be relevant in the long run [1-7].

\section{Bibliography}

1. Bonner JA., et al. "Radiotherapy plus cetuximab for squamouscell carcinoma of the head and neck". The New England Journal of Medicine 354.6 (2006): 567-578. 
2. Mehanna H., et al. "Radiotherapy plus cisplatin or cetuximab in low-risk human papillomavirus-positive oropharyngeal cancer (De-ESCALaTE HPV): an open-label randomised controlled phase 3 trial". Lancet 393 (2019): 51-60.

3. Chang JY., et al. "Stereotactic ablative radiotherapy versus lobectomy for operable stage I non-small-cell lung cancer: a pooled analysis of two randomised trials". Lancet Oncology 16 (2015): 630-637.

4. Bahig H., et al. "Surgery versus SABR for early stage non-small cell lung cancer: the moving target of equipoise". Journal of Thoracic Diseas 9 (2017): 953-956.

5. Wolf G., et al. "Larynx preservation with induction chemotherapy (CT) and radiation (XRT) in advanced laryngeal cancer final results of the VA Laryngeal Cancer Study Group Cooperative Trial". Proc ASCO 12 (1993): 277.

6. Kishan AU., et al. "Long-term Outcomes of Stereotactic Body Radiotherapy for Low-Risk and Intermediate-Risk Prostate Cancer". JAMA 2 (2019): e188006.

7. Resnick MJ., et al. "Long-Term Functional Outcomes after Treatment for Localized Prostate Cancer". The New England Journal of Medicine 368 (2013): 436-445.

\section{Volume 3 Issue 11 November 2019}

(C) All rights are reserved by Debnarayan Dutta., et al. 